\section{Darstellung von Tumoren der weiblichen Brust mit MR-Elastographie}

\begin{abstract}
Zusammenfassung. Ziel: Darstellung von Brusttumoren mit der MR-Elastographie. Methode: Mittels eines Oszillators werden niederfrequente mechanische Schallwellen in das Brustgewebe eingekoppelt. Die lokale Beschaffenheit der Welle wird durch die elastischen Eigenschaften des Gewebes definiert. Mit Hilfe einer bewegungs-sensitiven Spinechosequenz können diese Wellen in der Phase des MR-Bildes dargestellt werden. Diese Messungen werden im Folgenden dazu benutzt, die lokale Elastizitätsverteilung zu rekonstruieren. In-vivo-Messungen von drei Patientinnen mit malignen Tumoren der Brust wurden durchgeführt. Ergebnisse: Der Messaufbau wurde von allen Patientinnen akzeptiert und führte zu keinerlei Unannehmlichkeiten im Kontaktbereich der Haut mit dem Oszillator. Die eingebrachten mechanischen Wellen penetrierten die Mamma vollständig unter Einschluss der Axilla und der brustwandnahen Abschnitte. Alle Tumoren konnten in der MR-Elastographie lokalisiert werden und stellten sich härter als das umliegende Brustgewebe dar. Zusätzlich wurde bei einer Patientin in der MRElastographie eine axilläre Lymphknotenmetastase nachgewiesen. Bei allen Patientinnen stellten sich im übrigen Brustparenchym lokale Regionen erhöhter Elastizität dar (d.h. verhärtete Areale), ohne dass die Elastizitätswerte der Tumorregion erreicht wurden. Schlussfolgerung: Mit Hilfe der MRE ist eine ergänzende bildgebende Gewebscharakterisierung von Brusttumoren möglich.
\end{abstract}

Schlüsselwörter: MR-Elastographie - Brusttumor-Diagnostik Gewebecharakterisierung

Imaging of breast tumors using MR-Elastography. Purpose: Imaging of breast tumors using MR-Elastography. Material and method: Low-frequency mechanical waves are transmitted into breast-tissue by means of an oscillator. The local characteristics of the mechanical wave are determined by the elastic properties of the tissue. By means of a motion-sensitive spinecho-sequence these waves can be displayed within the phase of the MR image. Subsequently, these images can be used to reconstruct the local distribution of elasticity. In-vivo measurements were performed in 3 female patients with malignant tumors of the breast. Results: All patients tolerated the measurement set-up without any untoward sensation in the contact area of skin and oszillator. The waves completely penetrated the breast, encompassing the axilla and regions close to

Fortschr Röntgenstr 2001; 173: $12-17$

(c) Georg Thieme Verlag Stuttgart · New York

ISSN 1438-9029
J. Lorenzen ${ }^{1}$, R. Sinkus², D. Schrader ${ }^{3}$, M. Lorenzen ${ }^{3}$,

C. Leussler' ${ }^{2}$, M. Dargatz ${ }^{2}$, P. Röschmann ${ }^{2}$

${ }^{1}$ Abteilung für Röntgendiagnostik, Radiologische Klinik, Universitätskrankenhaus-Eppendorf, Hamburg

2 Philips Research Lab., Division Technical Systems, Hamburg

${ }^{3}$ Frauenklinik, Universitätskrankenhaus-Eppendorf, Hamburg

the chest wall. All tumors were localized by MRE as structures of markedly stiffer tissue when compared to the surrounding tissue. Furthermore, in one patient, a metastasis in an axillary lymph node was detetected. In all patients, local regions of increased elasticity were found in the remaining parenchyma of the breast, which, however, did not reach the high levels of elasticity found in the tumors. Conclusion: MRE is an imaging modality enabling adjunct tissue differentiation of mammary tumors.

Key words: MR-Elastography - Breast neoplasm diagnosis tissue characterisation

\section{Einleitung}

Das Mammakarzinom stellt in Deutschland und anderen hoch industrialisierten Ländern die mit Abstand häufigste Krebserkrankung der Frau dar. Sowohl die Morbidität als auch die Mortalität weist dabei eine steigende Tendenz auf [1]. Eine möglichst frühzeitige Diagnose kann jedoch die Heilungsaussichten wesentlich verbessern [2]. Durch die Mammographie und den Einsatz von ergänzenden Methoden wie Ultraschall, transkutanen Nadelbiopsien sowie kontrastmittelunterstützter MR-Mammographie konnten in der Mammadiagnostik hierbei entscheidende Fortschritte erreicht werden. Nichtsdestotrotz sind die diagnostischen Möglichkeiten begrenzt, denn selbst bei umfangreichen klinischen Untersuchungen ergeben sich immer wieder diagnostische Probleme bei der Malignomabklärung. So hat die Mammographie beispielsweise bei jungen Frauen mit dichtem Parenchym eine verminderte Sensitivität und Spezifizität. Die Einstufung von Läsionen in benigne und maligne ist häufig auch unter Einsatz der Sonographie nicht eindeutig sicher. Die kontrastmittelunterstützte MR-Mammographie stellt mittlerweile mit ihrer hohen Sensitivität zwar eine wichtige Ergänzungsmethode dar. Sie wird aber aufgrund ihrer eingeschränkten Spezifität nur für spezielle Fragestellungen empfohlen und ist als Screening-Verfahren nicht geeignet [3]. Zusammenfassend kann man feststellen, dass keines der erwähnten Verfahren eindeutig zwischen gutartigen Läsionen und malignen Gewebsveränderungen in der Mamma unterscheiden kann. Die Entwicklung neuer Bildgebungsverfahren zur Darstellung zusätzlicher Gewebe- 
parameter, die das Potenzial zur Erhöhung der Spezifität besitzen, ist daher sinnvoll und notwendig.

Die MR-Elastographie ist ein neuartiges Bildgebungsverfahren welches es ermöglicht, die In-vivo-Elastizitätsverteilung des Gewebes zu messen [4]. Generell stellt Palpation die erste Untersuchungsmodalität des Arztes bei der Suche nach pathologischen Veränderungen im Bereich der weiblichen Brust dar, d.h. Elastizität ist ein für den Arzt wichtiger Gewebeparameter. Signifikante Variationen der lokalen Gewebselastizität sind ein Hinweis für eine notwendige weitere klinische Abklärung. Aufgrund der somit großen Bedeutung dieses Parameters ist die Hoffnung berechtigt, dass die MR-Elastographie (MRE) als objektives Verfahren einen zusätzlichen Nutzen bei der Malignomabklärung darstellt. Ziel der Studie ist die Erprobung des neuartigen Messverfahrens an Patientinnen mit positivem Tumorbefund sowie die Darstellung der In-vivo-Elastizität der Tumoren.

\section{Material und Methode}

\section{Messmethode}

Bei der dynamischen MRE werden sinusförmige mechanische Wellen (Frequenz $v=65 \mathrm{~Hz}$ ) mittels eines Oszillators in das zu untersuchende Gewebe eingekoppelt. Die Eigenschaften der Welle, d.h. Amplitude und Phase, werden durch die lokalen elastischen Eigenschaften des Gewebes bestimmt. Eine Messung der Welle gestattet es somit, die lokale Elastizitätsverteilung zu rekonstruieren. Die Einkopplung der mechanischen Welle führt zu einer Vibration des penetrierten Gewebes in der Größenordnung von 30 - $200 \mu \mathrm{m}$. Dies ist ausreichend, um von einer bewegungssensitiven MR-Sequenz gemessen zu werden. Mit einer zur mechanischen Anregung phasenstabilen MRDatenakquisition zeigt dann die Phase des gemessenen MRBildes eine Momentaufnahme der sich bewegenden Welle innerhalb einer Schicht [5]. Aus diesen mit MR gemessenen Momentaufnahmen wird mit der nachgeschalteten Rekonstruktion schichtweise das Elastizitätsmodul errechnet [6].

\section{Messaufbau}

Abb.1 zeigt schematisch den Messaufbau sowie die MREBrücke. Die Patientin befindet sich in Bauchlage mit den Armen seitlich am Körper wobei beide Mammae frei nach unten hängen. Der die mechanischen Schwingungen erzeugende Oszillator wird seitlich leicht gegen eine der Mammae gedrückt. Er wird mittels eines Pulsgenerators und eines Verstärkers angetrieben, wobei diese wiederum vom Spektrometer des Kernspintomographen gesteuert werden. Die MREmpfangsspule besteht aus zwei einzelnen Synergiespulen. Jede Synergiespule besteht aus zwei zusammengeschalteten ringförmigen Einzelspulen. Jeweils zwei der Einzelspulen $(\varnothing$ $20 \mathrm{~cm}$ ) sind im oberen Bereich der Patientenbrücke brustwandnah und zwei Einzelspulen $(\varnothing 13 \mathrm{~cm})$ im unteren Bereich mamillennah positioniert. Der Synergiebetrieb erlaubt es entweder beide Synergiespulen gleichzeitig oder jeweils nur eine der Seiten auszulesen. Der Vorteil dieser entkoppelten Einzelspulen liegt im höheren SNR sowie in der geringeren Sensitivität gegenüber Flussartefakten.

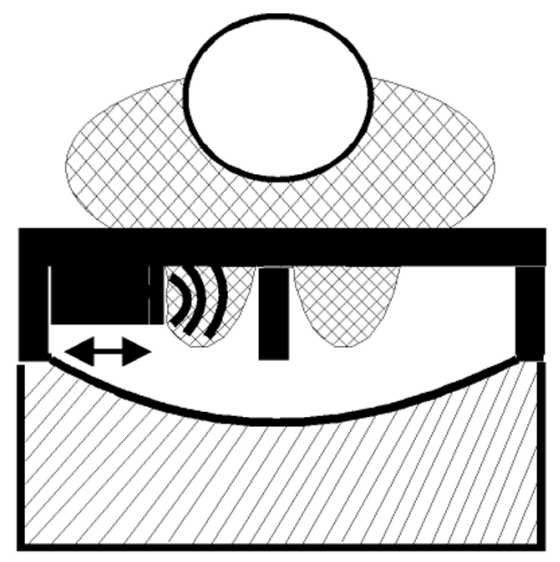

Abb. 1 (a) Messaufbau des MRE-Experimentes. Die Patientin befindet sich in Bauchlage, wobei der Oszillator seitlich an die zu untersuchende Brust leicht angedrückt wird. Dieser erzeugt niederfrequente Schallwellen (50 $100 \mathrm{~Hz}$ ) die in das Gewebe eindringen. Die MR-Empfangsspule ist hier nicht eingezeichnet. (b) Fotografie der Patientenbrücke mit Oszillator und Spule. Der Oszillator (Pfeil) ist verschieblich in die Brustspule integriert, um eine optimale Anpassung an die jeweilige Größe der Brust zu ermöglichen.

\section{Kernspintomograph und Pulssequenz}

Für die Untersuchungen wurde ein Standard Kernspintomograph Philips Gyroscan ACS-NT 1,5 T ausgerüstet mit dem Gradientensystem Powertrak 6000 verwendet. Die StandardProduktsoftware wurde dahingehend erweitert, dass neben trapezoidförmigen bewegungskodierenden Gradienten (wie z. B. bei diffusionsgewichteten MR-Sequenzen verwendet) auch sinusförmige Gradienten vom System realisiert werden. Die Repetitionszeit betrug $500 \mathrm{~ms}$ bei einer Echozeit von $45 \mathrm{~ms}$. Es wurde ein FOV von $140 \mathrm{~mm}$ bei einer Matrix von $128^{2}$ angewandt. Der zeitliche Ablauf der MR-Sequenz muss auf die Besonderheiten des MRE Experimentes abgestimmt werden. Bei einer mechanischen Anregungsfrequenz von $v=65 \mathrm{~Hz}$ muss die Bildgebungssequenz (hier eine Spinechosequenz) eine Repetitionszeit TR aufweisen, welche ein ganzzahliges Vielfaches der Anregungsfrequenz ist, d.h. TR $=\mathrm{N}^{*} \mathrm{~T}$ mit $\mathrm{T}=1000 / \mathrm{v}$ (ms) und N ganzzahlig. Dadurch ist die Bildgebung phasenstabil zur mechanischen Anregung. Dies ermöglicht es, die mechanische Welle zu einem bestimmten Zeitpunkt innerhalb der Schwingungsperiode abzubilden. In Abb. 2 ist die modifizierte Bildgebungssequenz des Kernspintomographen schematisch dargestellt. Einzige Änderungen gegenüber der Standard Spinechosequenz sind die sinusförmigen Gradienten vor und hinter dem 180-Grad-Puls. Die Gesamtmesszeit für 10 Schichten bei einer Schichtdicke von $2,5 \mathrm{~mm}$ und einem FOV von $140 \mathrm{~mm}$ beträgt $30 \mathrm{~min}$, da die Welle zu jeweils 8 unterschiedlichen Zeitpunkten sowie in alle drei Raumrichtungen gemessen werden muss. 


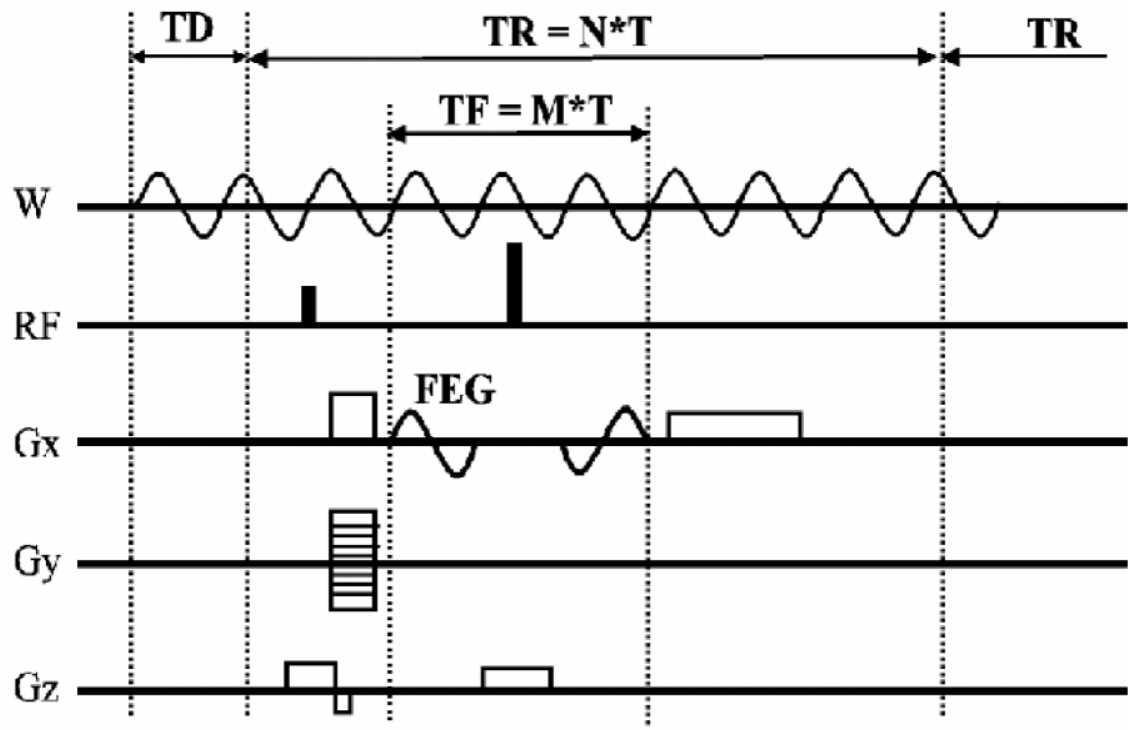

Abb. 2: MR-Sequenz des MRE-Experimentes: Eine Standard-Spinechosequenz wird durch sinusförmige flusskodierende Gradienten (FEG) erweitert. Die zusätzlichen Gradienten befinden sich zu beiden Seiten des refokussierenden 180-Grad-Pulses. Die Wahl des Gradientenkanals (Gx, Gy und Gz) bestimmt, welche räumliche Komponente der mechanischen Welle gemessen wird. Der zeitliche Ablauf der Sequenz ist dergestalt, dass sie phasenstabil zur mechanischen Anregung (W) ist. Die Länge der zeitlichen Verzögerung (TD) bestimmt, welcher Zustand (Phase) der mechanischen Welle durch das Bildgebungsexperiment gemessen wird. Eine Variation von TD ermöglicht die Messung der Bewegung eines jeden Voxels über die gesamte Schwingungsperiode.

\section{Rekonstruktion}

Eine ausführliche Darstellung der verwendeten Rekonstruktionsmethode ist in [6] beschrieben. Der bei diesem Verfahren entscheidende Fortschritt ist, dass für jeden gemessenen Bildpunkt der gesamte Elastizitätstensor rekonstruiert wird. Dies soll kurz erläutert werden: Normales elastisches Material wie z. B. Gummi hat eine Elastizität, die in alle Raumrichtungen gleich ist. Dies trifft beispielsweise auf Muskelgewebe nicht zu. Hier herrscht ein signifikanter Unterschied in der Härte, je nachdem in welche Raumrichtung gemessen wird, d.h. in Faserrichtung oder senkrecht zu dieser. Weist ein Material unterschiedliche Härten in den verschiedenen Raumrichtungen auf, so wird es als anisotrop bezeichnet. Diese Eigenschaft kann zur zusätzlichen Gewebecharakterisierung dienen. Die Darstellung der Elastizitäts- bzw. der Anisotropiewerte erfolgt anhand einer Farbskala.

\section{Patienten}

Es wurden drei Patienten mit vorgesehener Brust-OP eines palpablen Mammatumors präoperativ untersucht. Alle Patienten erhielten neben der klinischen Untersuchung eine Mammographie und eine Sonographie der Brust. Bei zwei Patientinnen erfolgte vor der MRE zusätzlich eine MR-Mammographie nach einem Standardprotokoll. Die MRE wurde nach entsprechender Aufklärung der Patientinnen durchgeführt. Die Lokalisation des Tumors und seine Ausdehnung wurden mit den Ergebnissen der Mammographie, Sonographie, MRT, sowie des OP-Berichts und des histologischen Befundes verglichen. Die Studie ist von der Ethikkommission der Hamburger Ärztekammer genehmigt.

\section{Ergebnisse}

Der Messaufbau wurde von allen Patientinnen akzeptiert und führte zu keinerlei Unannehmlichkeiten oder Beanstandungen. Ein Wärmegefühl oder Hautalterationen im Kontaktbereich zum Oszillator trat nicht auf. Die eingebrachten mechanischen Wellen penetrierten die Mamma vollständig unter Einschluss der Axilla und der brustwandnahen Abschnitte (Abb. 3 b). Die dabei erreichte Auflösung der MRE betrug bis zu 5 mm. Hierbei ist zu bemerken, dass die räumliche Auflösung der Methode nur indirekt durch die Frequenz der eingekoppelten Welle (und dadurch durch die von der Elastizität abhängenden Wellenlänge) begrenzt ist. Aufgrund der gewählten phasensensitiven Rekonstruktionsmethode [6] ist der wesentlich limitierende Faktor das SNR in der Messung. Die gewählte Frequenz von 65 $\mathrm{Hz}$ resultiert aus dem Kompromiss zwischen gewünschter kleiner Wellenlänge (also hoher Frequenz) und tiefer Penetration. Durch Dispersionseffekte bei der Dämpfung wird gerade die Penetration bei Erhöhung der Frequenz immer geringer, da die Dämpfung anwächst.

\section{Rekonstruktion}

Die Tumoren konnten in der MRE in ihrer Lokalisation abgegrenzt werden. Dabei stellte sich das Malignom in allen Fällen härter bzw. mit höherer Elastizität als das umliegende Brustgewebe dar. Zusätzlich wurde bei einer Patientin in der MRE eine axilläre Lymphknotenmetastase nachgewiesen (Abb.4 b). In den übrigen Anteilen des erfassten Brustparenchyms ließen sich Areale erhöhter Härte (Elastizität) abgrenzen, die jedoch unterhalb der Werte der Tumorregion lagen. Im Bereich der Auflagefläche der sternumnahen Brustwandanteile fanden sich bei allen Patientinnen erhöhte Elastizitätswerte, ohne dass sich mammographisch oder sonographisch verdichtetes Brustparenchym bzw. Tumoranteile nachweisen ließen. In der Darstellung der Anisotropie des Elastizitätsmoduls zeigte das Tumorgewebe grundsätzlich die höchste Anisotropie im Vergleich zum übrigen Brustparenchym (Abb. 5 c)

\section{Fall 1}

Bei einer 57-jährigen Patientin ließ sich im oberen äußeren Quadranten eine derbe Resistenz tasten. In der Mammographie ließen sich in gleicher Lokalisation zwei unmittelbar benachbarte Verdichtungszonen mit spikulaartigen Ausläufern von 2,9 $\mathrm{x} 2 \mathrm{~cm}$ und 1,5 x 1,2 cm nachweisen. Sonographisch bestätigten sich diese Herde als echoarme, unscharf begrenzte Herde. Auch in der MR-Mammographie bestätigten sich die beiden Herde als malignomsuspekt im Sinne einer vermehrten Kontrast- 

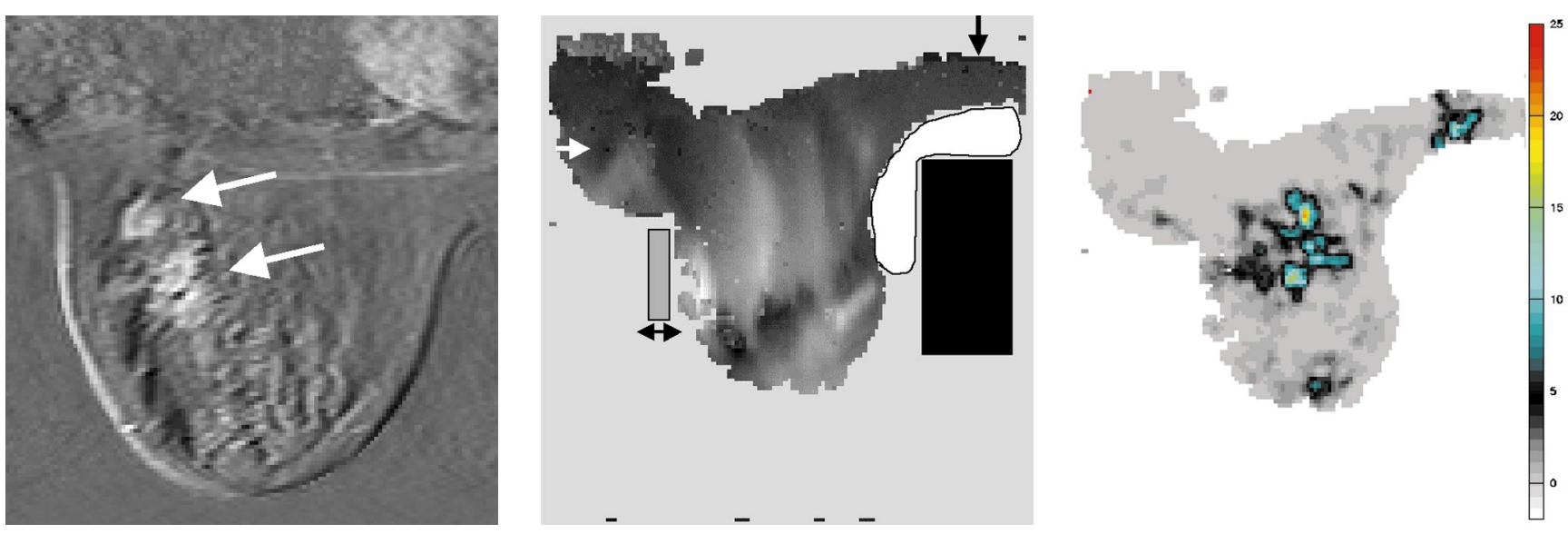

Abb. 3: Patientin mit einem invasiv-duktalen Karzinom im oberen äußeren Quadranten und einem unmittelbar angrenzenden Satellitenknoten. In (a) ist das Subtraktionsbild der Kontrastmittelserie der MRMammographie dargestellt. Es lassen sich zwei unmittelbar benachbarte Herde abgrenzen (Pfeile). In (b) ist das mit MRE gemessene Wellenbild der dazu korrespondierenden Schicht abgebildet. Der Oszillator wird von lateral gegen die Mamma gepresst (gräuliches Rechteck). Medial befindet sich die mit Schaumstoff (weiße Region) gepolsterte Auflagefläche (schwarzes Rechteck). Die Amplitude der mechanischen Welle ist durch Grauwerte dargestellt. Man erkennt deutlich eine gute Penetration bis in die Axillenregion (weißer Pfeil).

mittelanreicherung (Abb. 3 a). Die anschließend durchgeführte MRE wies ebenfalls in dieser Lokalisation zwei unmittelbar ineinander übergehende Herde deutlich erhöhter Härte nach (Abb.3c). Nach Ablatio mammae und Axillendissektion fand sich histologisch ein $3 \mathrm{~cm}$ großes, schlecht differenziertes Mammakarzinom, sowie $1 \mathrm{~cm}$ vom primären Herd entfernt ein Satellitenknoten von $1 \mathrm{~cm}$ Größe. Kein Nachweis von Lymphknotenmetastasen.

\section{Fall 2}

Bei einer 36-jährigen Patientin war ein rasch wachsender Tastbefund der linken Brust bei 12 Uhr aufgefallen. Mammographisch und sonographisch wurde ein malignomsuspekter Herd von 3,7 cm Größe nachgewiesen. Zusätzlich wurde in der Sonographie der Verdacht auf eine 1,9 cm große Lymphknotenmetastase geäußert. Im Rahmen einer Studie wurde bei der Patientin eine F18-FDG-Positronenemissionstomographie (PET) durchführt. Die PET zeigte neben einem vermehrten Glukosestoffwechsel in der Tumorregion eine gesteigerte lokale Glukoseaktivität links axillär, passend zu vitalem Tumorgewebe im Sinne einer Lymphknotenmetastase (Abb. 4 a). Als Korrelat in der MRE ließ sich axillär eine umschriebene Läsion erhöhter Elastizität nachweisen (Abb. 4 b). Die Axillenresektion ergab eine solitäre kapselinvasive Metastase des invasiv-duktalen Karzinoms.

\section{Fall 3}

Mammographisch und sonographisch ließ sich bei einer 56jährigen Patientin ein $3 \mathrm{~cm}$ großer malignomverdächtiger Herd im linken oberen Quadranten nachweisen. In die Axilla ziehend fanden sich spikulaartige Ausläufer mit polymorph strukturierten Mikroverkalkungen (Abb.5a). Die MRE zeigte in dieser Region eine polymorph strukturierte Läsion erhöhter Elastizität mit Ausläufern in die Axillaregion (Abb.5b). Im
Durch die Gewebskompression im Auflagebereich (Sternum, schwarzer Pfeil) wird dort lokal die Amplitude der Welle stark reduziert. In (c) ist das entsprechend rekonstruierte Elastogramm gezeigt. Mittels einer Farbskala werden die unterschiedlichen Härtegrade in Einheiten von $\mathrm{kPa}$ visualisiert. Entsprechend der MR-Mammographie werden zwei Herde deutlich erhöhter Elastizität im Vergleich zum umliegenden Mammagewebe dargestellt. Diese Herde kommen bedingt durch die laterale Kompression der Mamma medialisiert zur Darstellung. Die Verhärtung im Sternumbereich ist bedingt durch die Verhärtung des Gewebes aufgrund der Patientenlagerung.

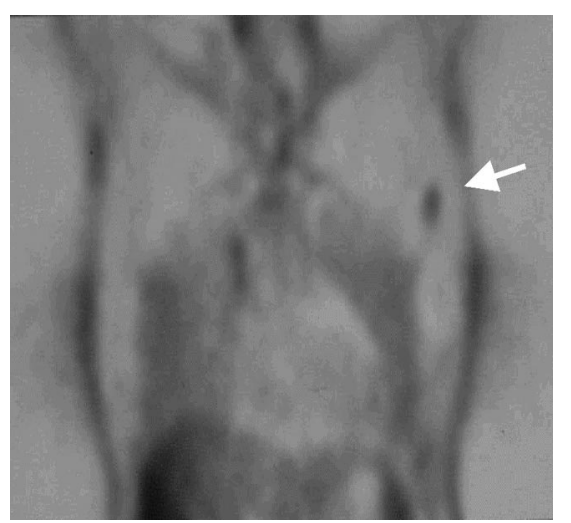
narer Schnitt des Körperstamms einer FDG-PET-Untersuchung mit Nachweis eines gesteigerten Glukosestoffwechsels in einer histologisch verifizierten Lymphknotenmetastase eines Mammakarzinoms (Pfeil).

(b) Im Elastogram ist axillär eine suspekte Region deutlich erhöhter Elastizität abzugrenzen (schwar-

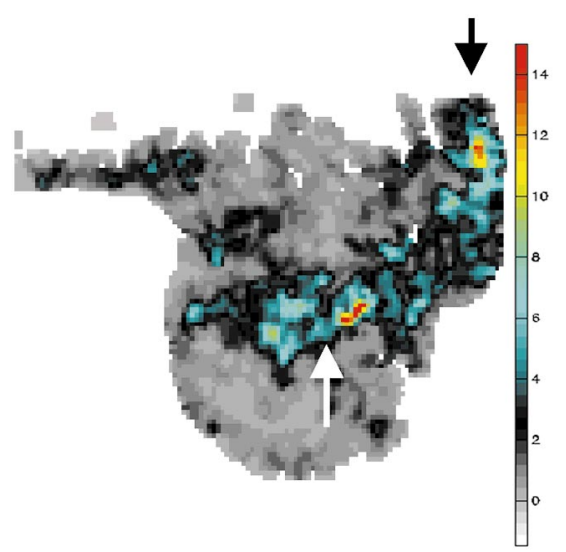
zer Pfeil). Der Tumor ist in dieser Schicht nur mit seinem oberen Anteil erfasst (weißer Pfeil).

Präparat wurde histologisch ein invasiv-duktales Karzinom von 2,8 cm Größe mit ausgeprägter Angioinvasion in die Tumorperipherie und Manifestationen eines high-grade DCIS nachgewiesen.
Abb. 4 (a) Koro- 

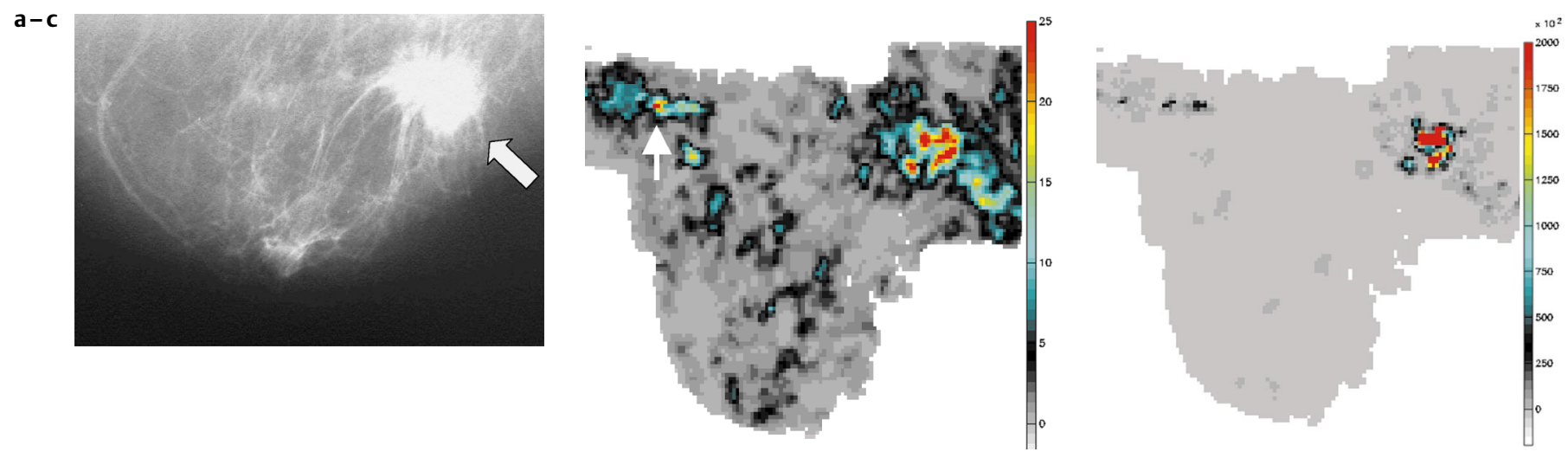

Abb.5 (a) Mammographieaufnahme eines invasiv-duktalen Karzinoms im oberen äußeren Quadranten mit Nachweis eines unscharf begrenzten Herdbefundes mit Spikulae (Pfeil) in die axilläre Tumorperipherie. (b) Korrespondierendes Elastogramm. Nachweis des Tumors mit Ausläufern in die Axillenregion. Zusätzlich finden sich weitere

\section{Diskussion}

Grundlage der vorliegenden Arbeit ist der physikalische Parameter der Elastizität, ein Parameter der jedem klinisch tätigen Arzt vertraut ist. Pathologische Veränderungen im Gewebe wie z.B. Tumoren sind häufig durch Veränderung der Elastizität innerhalb des gesunden Gewebes abgrenzbar. Daher stellt die Abtastung der weiblichen Brust eine wichtige diagnostische Verteidigungslinie beim Brustkrebs dar. Allerdings ist die Palpation limitiert durch die Größe der Läsion, ihre Lokalisation im umgebenden Gewebe und die subjektive Einschätzung des Untersuchers.

Diagnostische Verfahren zur Bestimmung der räumlichen Verteilung der Elastizität sind ein neuer Ansatzpunkt der medizinischen Forschung. Das mögliche klinische Potential der Elastizität konnten Garra et al. für die sonographische Elastographie bei Patientinnen mit abklärungsbedürftigen Herden in der Brust aufzeigen [7]. Nachteil der sonographischen Elastographie ist die Einschränkung der Darstellbarkeit auf zwei Raumdimensionen mit einer akzeptablen Auflösung nur in axialer Ausrichtung [8]. Diese Einschränkungen gelten für die MR-Elastographie nicht. Hier haben sich zwei unterschiedliche Methoden zur Messung der Elastizität entwickelt. Bei der so genannten dynamischen MRE werden sinusförmige mechanische Wellen mittels eines Oszillators in das zu untersuchende Gewebe eingekoppelt. Die Eigenschaften der Welle (d.h. Amplitude und Phase) werden durch die lokalen elastischen Eigenschaften des Gewebes bestimmt [5,9]. Eine Messung der Welle erlaubt somit die Berechnung der Elastizität. Der zweite Ansatz hingegen verwendet eine statische Kompression und Dekompression des Gewebes [10]. Aus der mit MR gemessenen Verschiebung der einzelnen Voxel kann dann die lokale Elastizitätsverteilung rekonstruiert werden.

Voraussetzung für die Messung des Elastizitätsmoduls beim dynamischen Ansatz der MRE ist eine ausreichende Penetration der mechanischen Wellen. In der vorliegenden Studie fand sich bei allen drei Patientinnen eine gute Einkopplung der Welle in das Mammagewebe mit einer vollständigen Penetration der Wellen bis in die Axilla. Bei Patient 2 ließ sich daher eine Lymphknotenmetastase in der Axilla nachweisen. Bishop
Regionen vermehrter Elastizität, insbesondere sternumnah (Pfeil), die allerdings nicht die Werte der Tumorregion erreichen. (c) In der Darstellung der Elastizität gewichtet nach ihrer Anisotropie weist die Tumorregion ein hohes Ausmaß an Anisotropie auf, während sämtliche andere Regionen isotrop (grau) erscheinen.

et al. wiesen bereits auf das Problem einer nicht ausreichenden Penetration in der MRE bei humanem Gewebe hin [9]. Allerdings erfolgte in dieser Studie die Einkopplung der mechanischen Wellen durch transversale und nicht durch longitudinale Anregung wie in der vorliegenden Arbeit. Auf die Vorteile der longitudinalen Anregung für eine suffiziente Penetration der Welle bei valider Wiedergabe des Elastizitätsmoduls wurde in [6] hingewiesen.

Die bisher publizierten In-vivo- und In-vitro-Daten zur MRElastographie beschreiben eine Abgrenzbarkeit des Tumors gegenüber dem umliegenden Gewebe aufgrund seiner erhöhten Elastizität [11-13]. Auch bei unseren Patientinnen stellte sich die Tumorregion signifikant härter als das umliegende Gewebe dar. Allerdings fanden sich bei allen Patientinnen auch innerhalb des übrigen mitdargestellten Brustgewebes umschriebene Areale erhöhter Elastizität, die jedoch nicht die Werte der Tumorregion erreichten. Inwieweit eine sichere Trennung auch primär nicht palpabler Tumoren durch die MRE von z.B. umliegendem mastopathischen Gewebe möglich ist, muss erst an größeren Patientenkollektiven untersucht werden. Ebenso ist bisher nicht nachgewiesen, ob die MRE eine Hilfestellung bei der Differenzierung von benignen und malignen Raumforderungen der Brust geben kann. Für die sonographische Elastographie konnte allerdings bereits der Einsatz der Elastographie in der Differenzierung maligner und benigner Raumforderungen von Brusttumoren als eine hilfreiche Ergänzung gezeigt werden [7].

Der in der vorliegenden Arbeit gewählte Messaufbau und Rekonstruktionsmodus ermöglicht erstmals die korrekte Bestimmung des Elastizitätmoduls in allen drei Raumrichtungen, d.h. es wird der symmetrische Elastizitätstensor ermittelt. Es ist daher möglich, die lokale Elastizität gewichtet entsprechend ihrer Anisotropie darzustellen. Von biologischem Gewebe ist bekannt, dass es im Gegensatz zu künstlichen Substanzen (z. B. Gummi) in unterschiedlicher Ausprägung anisotrop ist [14]. Die ausgeprägte Anisotropie der Tumoren in unserer Studie (Abb. 5c), die sich von dem übrigen Brustparenchym unterscheidet, ist möglicherweise ein weiterer Parameter in der Gewebecharakterisierung durch die MRE. Die Ursache der erhöhten Anisotropie könnte in dem häufig heterogenen 
Aufbau von Tumoren liegen. Weitere Untersuchungen sind nötig, um diese Hypothese zu überprüfen.

Bei allen Patientinnen wies das sternumnahe Brustgewebe erhöhte Elastizitätwerte auf, ohne Nachweis eines pathomorphologischen Korrelats in den übrigen Untersuchungen. Diese Region der Brust wird bedingt durch die Bauchlage der Patientin und den gewählten Messaufbau verstärkt komprimiert was zu signifikant erhöhten Werten der Elastizität führt, die eine valide Beurteilung dieser Region zur Zeit nicht möglich machen. Änderungen in der Auflagefläche z.B. mit einer entsprechenden Polsterung könnten hier möglicherweise eine bessere Beurteilung ermöglichen. Vorteil der gewählten Lagerung der Patientin und des Messaufbaus ist die optionale Durchführung einer MR-Mammographie mit ergänzender MRE in einem Untersuchungsgang ohne notwendige Umlagerung der Patientin. Suspekte bzw. fragliche Befunde der MRMammographie könnten somit durch den Befund der MRE ergänzt werden.

Die Akquisitionszeit in unserer Studie betrug für einen Untersuchungsbereich von $2,5 \mathrm{~cm} 30$ Minuten. Eine gleichzeitige Durchführung einer MR-Mammographie und ergänzenden MR-Elastographie mit einer Gesamtmesszeit von nahezu 1 Stunde ist klinisch nicht praktikabel. Nichtsdestotrotz ist der gewählte Messaufbau je nach klinischer Anforderung erweiterbar in zwei Richtungen. Durch Anwendung einer GradientenEcho-Sequenz und Reduzierung der Messwerte pro Pixel von zur Zeit 8 Zeitpunkten der Schwingungsperiode auf 4 Messpunkte der Wellen-Konfiguration ist eine Untersuchung der gesamten Brust mit MRE in 30 Minuten möglich. Bei einer Kombination der MR-Mammographie mit der MRE in einem Untersuchungsgang ist die Beschränkung auf Brustläsionen möglich, die eine signifikante Kontrastmittelanreicherung aufweisen. Durch Reduzierung des FOV auf z.B. $64 \mathrm{~mm}$ und einer Matrix von $64^{2}$ in der MRE ist eine ergänzende Bestimmung der Elastizität und Anisotropie einer solchen Läsion in ca. 7 Minuten durchführbar.

\section{Schlussfolgerungen}

Die Ergebnisse dieser Studie zeigen, dass es mit dem entwickelten Messaufbau möglich ist, maligne Tumore der weiblichen Brust mit Hilfe der MRE darzustellen und vom übrigen Parenchym abzugrenzen. Inwieweit eine Unterscheidung von benignen und malignen Tumoren und einer Detektion kleiner Tumoren unter $1 \mathrm{~cm}$ erreichbar ist, muss die weitere klinische Forschung zeigen. Die Möglichkeit einer ergänzenden bildgebenden Gewebecharakterisierung durch die MRE könnte zu einer Steigerung der Spezifität der MR-Mammographie in einem Untersuchungsgang beitragen.

\section{Literatur}

${ }^{1}$ Becker N, Wahrendorf J. Krebsatlas der Bundesrepublik Deutschland 1981 - 1990.; 3. Auflage Berlin. Springer 1998

${ }^{2}$ Carter CL, Allen C, Henson DE. Relation of tumor size, lymph node status, and survival in 24740 breast cancer cases. Cancer 1989; 63 : $181-187$

${ }^{3}$ Heywang-Köbrunner S. Contrast-Enhanced Magnetic Resonance Imaging of the breast. Invest. Radiology 1994; 29: 94-104

${ }^{4}$ Muthupillai R, Lomas DJ, Rossman PJ, Greenleaf JF, Manduca A, Ehman RL. Magnetic Resonance Elastography By Direct Visualization Of Propagating Acoustic Strain Waves. Science 1995; 26: $854-1857$

${ }^{5}$ Muthupillai R, Prossman PJ, Lomas DJ, Greenleaf JF, Riederer SJ, Ehman RL. Magnetic Resonance Imaging Transverse Acoustic Strain Waves. MRM 1996; 36: 266 - 274

${ }^{6}$ Sinkus R, Lorenzen J, Schrader D, Lorenzen M, Dargatz M, Holz D. High resolution tensor MR-elastography for breast tumor detection. Phys. Med. Biol 2000; 45 Nr. 6: 1649-1664

${ }^{7}$ Garra BS, Cesdpedes EI, Ophir J, Spratt SR, Zuurbier RA, Magnant CM, Pennannen MF. Elastography of breast lesions: Initial clinical results. Radiology 1997; 202: 79-86

${ }^{8}$ Skovoroda A, Emelianov S, Lubinski M, Sarvazyan A, O’Donnell M. Theoretical analysis and verification of ultrasound displacement and strain imaging. IEEE Trans Ferro Freq Ctrl 1994; 41: 302 - 313

${ }^{9}$ Bishop J, Poole G, Leitch M, Plewes D. Magnetic resonance Imaging of Shear wave Propagation in Excised Tissue. J. Magn. Reson. Imaging 1998; 8: 1257-1265

${ }^{10}$ Chenevert TL, Skovoroda AR, O'Donnell M, Emelianov SY. Elasticity Reconstructive Imaging by Means of Stimulated Echo MRI. MRM 1998; 39: $482-490$

${ }^{11}$ Lawrence A, Rossman P, Mahowald J, Manduca A, Hartmann L, Ehman R. Proc. ISMRM $7^{\text {th }}$. Philadelphia. 1999: 525

12 Dresner M, Rossman P, Kruse S, Ehman R. Proc. ISMRM $7^{\text {th }}$. Philadelphia. 1999: 258

13 Sinkus R, Lorenzen J, Schrader D, Lorenzen M, Dargatz M, Holz D. Proc. ISMRM $7^{\text {th }}$. Philadelphia. 1999: 259

${ }^{14}$ Fung Y. Biomechanics, Mechanical Properties of Living tissues. Berlin. Springer 1993

Dr. Jörn Lorenzen

Radiologische Klinik

Univ.-Klinik Hamburg-Eppendorf

Martinistraße 52

20246 Hamburg

Tel. 040-42803-3527

Fax 040-42803-4759 